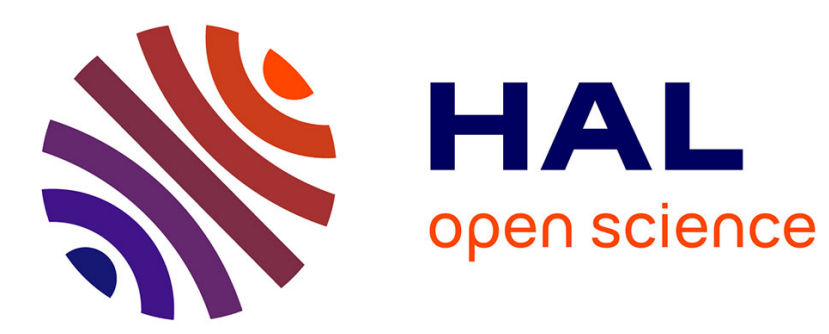

\title{
All-to-All Routing and Coloring in Weighted Trees of Rings
}

Bruno Beauquier, Stéphane Pérennes, David Tóth

\section{To cite this version:}

Bruno Beauquier, Stéphane Pérennes, David Tóth. All-to-All Routing and Coloring in Weighted Trees of Rings. RR-3700, INRIA. 1999. inria-00072968

\section{HAL Id: inria-00072968 \\ https://hal.inria.fr/inria-00072968}

Submitted on 24 May 2006

HAL is a multi-disciplinary open access archive for the deposit and dissemination of scientific research documents, whether they are published or not. The documents may come from teaching and research institutions in France or abroad, or from public or private research centers.
L'archive ouverte pluridisciplinaire HAL, est destinée au dépôt et à la diffusion de documents scientifiques de niveau recherche, publiés ou non, émanant des établissements d'enseignement et de recherche français ou étrangers, des laboratoires publics ou privés. 
INSTITUT NATIONAL DE RECHERCHE EN INFORMATIQUE ET EN AUTOMATIQUE

\section{All-to-All Routing and Coloring in Weighted Trees of Rings}

Bruno Beauquier — Stéphane Pérennes — David Tóth

$\mathbf{N}^{\circ} 3700$

Juin 1999

THÈME 1 



\title{
All-to-All Routing and Coloring in Weighted Trees of Rings
}

\author{
Bruno Beauquier * $^{*}$, Stéphane Pérennes * ${ }^{*}$ David Tóth ${ }^{\dagger}$ \\ Thème 1 - Réseaux et systèmes \\ Projet Sloop
}

Rapport de recherche $\mathrm{n}^{\circ} 3700$ - Juin $1999-15$ pages

\begin{abstract}
A tree of rings is an undirected graph obtained from the union of rings, which intersect two by two in at most one node, such that any two nodes are connected by exactly two edge-disjoint paths. In this paper, we consider symmetric directed trees of rings with weighted nodes. A routing for a weighted digraph is a collection of directed paths (dipaths), such that for each ordered pair of nodes $\left(x_{1}, x_{2}\right)$ with respective weights $w_{1}$ and $w_{2}$, there are $w_{1} w_{2}$ dipaths (possibly not distinct) from $x_{1}$ to $x_{2}$. Motivated by the Wavelength Division Multiplexing (WDM) technology in all-optical networks, we study the problem of finding a routing which can be colored by the fewest number of colors so that dipaths of the same color are arc-disjoint. We prove that this minimum number of colors (wavelengths) is equal to the maximum number of dipaths that share one arc (load), minimized over all routings. The problem can be efficiently solved (dipaths found and colored) using cut properties.
\end{abstract}

Key-words: optical networks, WDM, routing, coloring, cut.

Accepted in the 11th ACM Symposium on Parallel Algorithms and Architectures (SPAA'99).

This work has been partially supported by the AFIRST in the framework of the French-Israeli project Communication Algorithms in Optical Networks and by the French RNRT project PORTO.

* SLOOP is a joint project with the CNRS and the University of Nice-Sophia Antipolis (I3S laboratory). E-mail: \{Bruno.Beauquier,Stephane.Perennes\}@inria.fr.

$\dagger$ Technical University of Budapest, research done while visiting INRIA at Sophia Antipolis. E-mail: tocsa@math.bme.hu. 


\section{Routage Optique WDM dans les arbres d'anneaux pondérés}

Résumé : Un arbre d'anneaux est un graphe non-orienté obtenu par l'union d'anneaux, qui s'intersectent deux-à-deux en au plus un sommet, de telle sorte que deux sommets sont reliés par exactement deux chemins arête-disjoints. Dans ce rapport, nous considérons des arbres d'anneaux orientés symétriques avec des sommets pondérés. Un routage pour un graphe orienté pondéré est une collection de chemins orientés, telle que pour tout couple de sommets $\left(x_{1}, x_{2}\right)$ de poids respectifs $w_{1}$ et $w_{2}$, elle contient $w_{1} w_{2}$ chemins (éventuellement distincts) allant de $x_{1}$ vers $x_{2}$. Motivés par la technologie de multiplexage en longueurs d'onde WDM (Wavelength-Division Multiplexing) dans les réseaux tout-optiques, nous étudions le problème de trouver un routage et une coloration minimale des chemins, avec la contrainte que deux chemins de la même couleur sont arc-disjoints. Nous montrons que le nombre minimum de couleurs (longueurs d'onde) pour une solution est égal au nombre maximum de chemins qui partagent un arc (charge), parmi tous les routages possibles. Le problème est résolu par une méthode constructive et efficace, en utilisant des critères de coupe.

Mots-clés : réseaux optiques, WDM, routage, coloration, coupe. 


\section{Introduction and Motivation}

Optics is emerging as a key technology in communication networks, promising very high speed local or wide area networks of the future. A single optical wavelength supports rates of gigabits per second, which in turn support multiple channels of voice, data and video $[10,13]$. Multiple laser beams that are propagated over the same fiber on distinct optical wavelengths can increase this capacity even further. This is achieved through Wavelength Division Multiplexing (or WDM) [6], by partitioning the optical bandwidth into a number of channels and allowing the transmission of multiple data streams concurrently along the same optical fiber on different channels, i.e., different wavelengths. (Two streams of the same wavelength cannot be routed on the same fiber due to electromagnetic interference.)

All-optical (or single-hop [14]) communication networks provide all source-destination pairs with end-to-end transparent channels that are identified through a wavelength and a physical path. They exploit photonic technology for the implementation of both the switching and the transmission functions [9], and maintain the signal in optical form through the transmission, thus allowing much higher data transmission rates (since there is no prohibitive overhead due to conversions to and from the electronic form). Wavelengths being a limited resource, solutions to the problem of efficient routing and wavelengths allocation are of importance for the future development of optical technology.

The problem we consider here is motivated by switched networks with reconfigurable wavelength selective optical switches, without wavelength converters, where different signals may travel on the same communication link (but on different wavelengths) into a node, and then exit from it on different links, keeping their original wavelengths. Since optical links are inherently bidirectional, the problem has initially been modeled by undirected graphs, as in $[1,2,15]$. However, it has since become clear that the use of amplifiers will actually make each bidirectional optical link into a pair of unidirectional links [16], and hence the new models of the situation tend to represent optical networks by symmetric directed graphs, as in $[5,12,11]$.

In an all-optical network one needs to set up a number of communications (dipaths) between given pairs of nodes, with each communication being transmitted on one particular wavelength, and so that all communications that share a link have different wavelengths. Specifically, the general problem is the following : Given a collection of requests $\left(x_{1}, y_{1}\right),\left(x_{2}, y_{2}\right), \ldots,\left(x_{k}, y_{k}\right)$, connect each $x_{i}$ to the corresponding $y_{i}$ by a dipath $P_{i}$, and assign a wavelength to each $P_{i}$, so that dipaths with the same wavelength do not share a link. The objective is to use the minimum possible number of wavelengths. This parameter is considered of importance in evaluating the competitiveness of the wavelength division multiplexing technology [12]. A survey of graph theoretic problems associated with routing in optical networks can be found in [4].

The problem of minimizing the number of wavelengths has been proved to be NPcomplete for a general collection of requests, even in rings [7]. Here we consider the All-To-All communication process in the weighted case : for each ordered pair of nodes $(x, y)$ with respective weights $w(x)$ and $w(y), w(x) w(y)$ dipaths (possibly not distinct) from $x$ to $y$ have to be found and assigned wavelengths. Optimal results for this problem have been proved in

$\mathrm{RR} \mathrm{n}^{\circ} 3700$ 
the unweighted case for rings [5, 18], for some cartesian products [3] including hypercubes and some toroidal meshes, for some compound graphs [17] and for trees [8].

In this paper we solve the case of the weighted symmetric directed trees of rings. These networks are interesting since tree-like structures are often used in the telecommunications industry [12] and emerging SDH (Synchronous Digital Hierarchy) local area networks generally consist of SONET (Synchronous Optical NETwork) rings connected together.

\section{Preliminaries}

In this paper, we model an all-optical network as a weighted symmetric digraph $(G, w)$, that is a directed graph $G$, with node set $V(G)$ and arc set $A(G)$ (such that if $(u, v) \in A(G)$ then $(v, u) \in A(G))$, together with a non negative integral weight function $w$ on the set of nodes. We always denote by $N$ the total weight $\sum_{v \in V(G)} w(v)$ of $(G, w)$, and for any subset of nodes $S \subset V(G)$ we define the weight of $S$ as $w(S)=\sum_{s \in S} w(s)$.

Definition $1 \mathrm{~A}$ tree of rings is an undirected graph obtained from the union of rings, which intersect two by two in at most one node, such that any two nodes are connected by exactly two edge-disjoint paths.

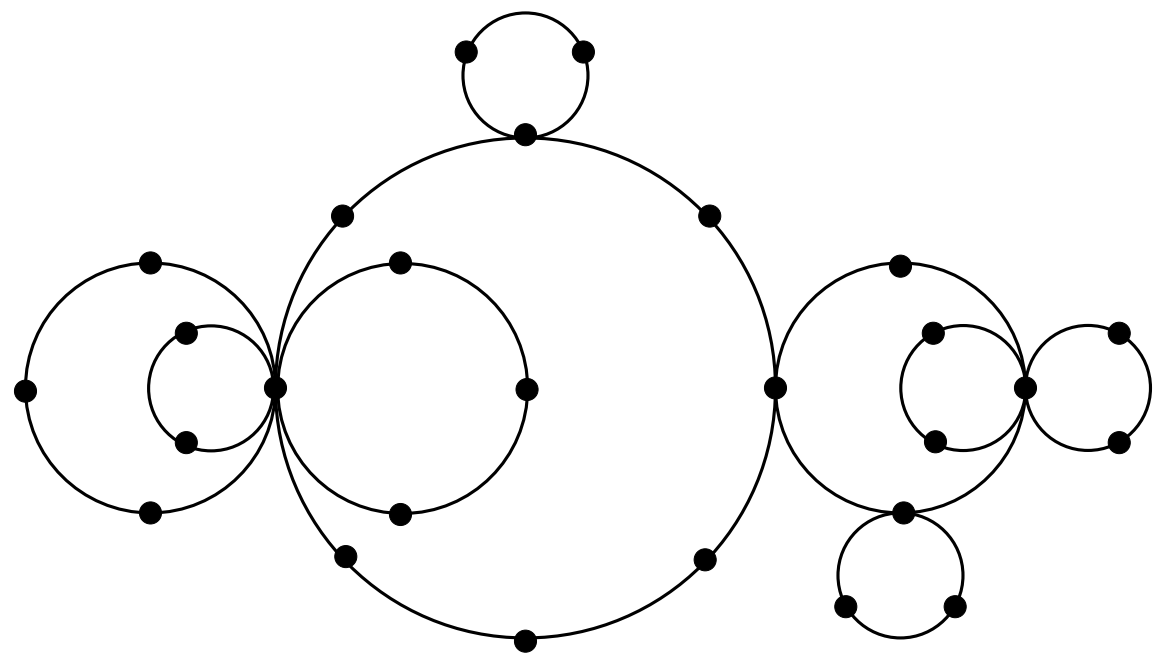

Figure 1: A tree of rings.

A symmetric digraph is naturally induced by an undirected graph by replacing each edge by two opposite arcs. In this paper, we consider weighted symmetric directed trees of rings. 
Definition 2 A routing for a weighted digraph $(G, w)$ is a collection of directed paths (dipaths) in $G$, such that for each ordered pair of nodes $(x, y)$ there are $w(x) w(y)$ dipaths (possibly not distinct) from $x$ to $y$.

The wavelength-routing problem for a weighted digraph $(G, w)$ consists of finding a routing and assigning each dipath a wavelength (or color), so that no two dipaths sharing an arc have the same wavelength. We denote by $\overrightarrow{\mathrm{w}}(G, w)$ the minimum number of wavelengths used to solve the wavelength-routing problem in $(G, w)$.

Given a weighted digraph $(G, w)$ and a routing $R$, the load of an arc $\alpha \in A(G)$ is the number of dipaths in $R$ containing $\alpha$. The load of $(G, w)$, denoted by $\vec{\pi}(G, w)$, is the minimum over all routings $R$ of the maximum load of the $\operatorname{arcs}$ of $G$. Clearly, $\vec{\pi}(G, w)$ is a lower bound on $\overrightarrow{\mathrm{w}}(G, w)$.

Property $3 \overrightarrow{\mathrm{w}}(G, w) \geq \vec{\pi}(G, w)$ for any weighted digraph $(G, w)$.

Given a weighted digraph $(G, w)$ and any partition $(S, \bar{S})$ of the set of nodes $V(G)$, called a $c u t$, we denote by $c(S, \bar{S})$ the number of arcs beginning in $S$ and ending in $\bar{S}$.

At least $w(S) w(\bar{S})$ dipaths in any routing for $(G, w)$ must use these arcs. So we have $\vec{\pi}(G, w) \geq \frac{w(S) w(\bar{S})}{c(S, \bar{S})}$ for any cut $(S, \bar{S})$.

As far as a weighted tree of rings $(\mathcal{T}, w)$ is concerned, we consider only cuts $(S, \bar{S})$ such that $c(S, \bar{S})=2$. Therefore $S$ and $\bar{S}$ are two connected components of $\mathcal{T}$. Setting $M=w(S)$ and $m=w(\bar{S})$, we have $M+m=N$ and we assume that $M \geq m$ in the sequel. The product $M m$ is called the load of the cut and the difference $\delta=M-m$ is called the gap of the cut. A tight cut is a cut with maximum load (or equivalently with minimum gap).

Given two nodes $a$ and $b$ of $(\mathcal{T}, w)$ with positive weights, we denote by $w_{a, b}$ the weight function obtained from $w$ by decreasing the weights of nodes $a$ and $b$ by one unit (hence $\left(\mathcal{T}, w_{a, b}\right)$ has total weight $\left.N-2\right)$. The minimum gap of $\left(\mathcal{T}, w_{a, b}\right)$ is denoted by $\delta_{a, b}$. The following property will be usefull :

Property 4 Let $(\mathcal{T}, w)$ be a weighted tree of rings with minimum gap $\delta \geq 2$. Let $a$ and $b$ be two nodes in $V(\mathcal{T})$ with positive weights. If there exists a tight cut $(S, \overline{\bar{S}})$ of $(\mathcal{T}, w)$ such that $a, b \in S$ then $\delta_{a, b}=\delta-2$, otherwise $\delta_{a, b} \geq \delta$.

Proof. Consider any cut $(S, \bar{S})$ of $\mathcal{T}$. If $(S, \bar{S})$ is a tight cut of $(\mathcal{T}, w)$, then $(S, \bar{S})$ has gap $\delta-2 \geq 0$ in $\left(\mathcal{T}, w_{a, b}\right)$ if $a, b \in S$, and gap at least $\delta$ in $\left(\mathcal{T}, w_{a, b}\right)$ if not. If $(S, \bar{S})$ is not a tight cut of $(\mathcal{T}, w)$, then it has gap at least $\delta+2$ in $(\mathcal{T}, w)$ and gap at least $\delta$ in $\left(\mathcal{T}, w_{a, b}\right)$.

Half the load of a tight cut is clearly a lower bound on both $\vec{\pi}(\mathcal{T}, w)$ and $\overrightarrow{\mathrm{w}}(\mathcal{T}, w)$. In this paper, we show that this bound is indeed exact :

Main Theorem For any weighted tree of rings $(\mathcal{T}, w)$ we have $\overrightarrow{\mathrm{w}}(\mathcal{T}, w)=\vec{\pi}(\mathcal{T}, w)=\left\lceil\frac{M m}{2}\right\rceil$, where $M m$ is the load of a tight cut.

The proof is done by induction on the total weight. We first prove the result in the case of weighted rings and then proceed with the general case.

$\mathrm{RR} \mathrm{n}^{\circ} 3700$ 


\section{Weighted rings}

In this section we prove the theorem in the case of weighted rings. The proof for arbitrary weighted trees of rings will follow the same lines.

Due to the upper integral part appearing in Main Theorem, we need to introduce the notion of half-colors. Any color assigned to a set of arc-disjoint dipaths can be seen as the union of two opposite half-colors : a positive one for clockwise dipaths and a negative one for anti-clockwise dipaths. Conversely, any two opposite half-colors can give rise to one color. Therefore, half-coloring a routing with $h^{+}$positive half-colors and $h^{-}$negative half-colors leads to a coloring with $\max \left\{h^{+}, h^{-}\right\}$colors. Half-colors for a routing are said to be balanced if $\left|h^{+}-h^{-}\right| \leq 1$. We will manage to have $h^{+}=\left\lceil\frac{M m}{2}\right\rceil$ and $h^{-}=\left\lfloor\frac{M m}{2}\right\rfloor$, so that we can get a solution to the wavelength-routing problem using a total of $\max \left\{h^{+}, h^{-}\right\}=\left\lceil\frac{M m}{2}\right\rceil$ colors. The following proposition implies Main Theorem for weighted rings.

Proposition 5 Let $(C, w)$ be a weighted ring and $M m$ be the load of a tight cut. There exists a routing that can be colored with $h^{+}=\left\lceil\frac{M m}{2}\right\rceil$ and $h^{-}=\left\lfloor\frac{M m}{2}\right\rfloor$ half-colors.

Our proof is by induction on the total weight $N$ : a coloring for $(C, w)$ is obtained from a coloring of the weighted ring $\left(C, w_{a, b}\right)$ for some two nodes $a$ and $b$.

Definition 6 Let $(C, w)$ be a weighted ring with total weight $N$. Given two nodes $a$ and $b$ with positive weights, let $A$ and $B$ be the two connected sets of nodes of $C-\{a, b\}$. Then nodes $a$ and $b$ are said to be antipodal if $w(A) \leq\lfloor N / 2\rfloor$ and $w(B) \leq\lfloor N / 2\rfloor$.

Intuitively, antipodal nodes can be seen in the following way. Assume that the nodes of the weighted ring are labelled with disjoint intervals of integers modulo $N$, so that each node $a$ has an interval $I(a)$ of length equal to $w(a)$ and adjacent nodes have adjacent intervals. Then, any two nodes $a$ and $b$ are antipodal if there are $x \in I(a)$ and $y \in I(b)$, such that $y=x+\left\lfloor\frac{N}{2}\right\rfloor \bmod N$ or $y=x+\left\lceil\frac{N}{2}\right\rceil \bmod N$.

\section{Case A}

Keeping the same notation as in Property 4, we assume that :

Condition A There exist two antipodal nodes $a$ and $b$ such that $\delta_{a, b} \geq \delta$.

Note that Condition A is fulfilled if $\delta \leq 1$, or if there exist two antipodal nodes $a$ and $b$, such that for any tight cut $(S, \bar{S}), a \in S$ if and only if $b \in \bar{S}$.

Lemma 7 If Proposition 5 holds for all weighted rings with weight less than $N$, then it holds for any weighted ring with weight $N$ satisfying Condition A.

Proof. Let $(C, w)$ be a weighted ring with weight $N$ satisfying Condition $\mathrm{A}$. (The result is obviously true if $N \leq 1$.) According to Condition A, there are two nodes $a$ and $b$ such that the load of a tight cut of $\left(C, w_{a, b}\right)$ is at most $(M-1)(m-1)=M m-(m+M)+1$. Using the inductive hypothesis, we color a routing of $\left(C, w_{a, b}\right)$ with $M m-(N-1)$ balanced 
half-colors. Let $A$ and $B$ be the two connected sets of nodes of $C-\{a, b\}$. To obtain a colored routing for $(C, w)$ satisfying the lemma, it remains to assign in a balanced way $N-1$ half-colors to a set $D_{1}$ of $4(w(A)+w(B))$ dipaths connecting $a$ and $b$ with the nodes in $A$ and $B$ and to a set $D_{2}$ of $2(w(a)+w(b)-1)$ dipaths connecting $a$ with $b$.

Given $a^{\prime} \in A \cup\{a, b\}$ and $b^{\prime} \in B \cup\{a, b\}$, two half-colors (one in each direction) are sufficient to color eight dipaths (possibly less if $a^{\prime} \in\{a, b\}$ or $b^{\prime} \in\{a, b\}$ ) connecting $a$ and $b$ with $a^{\prime}$ and $b^{\prime}$ (see Figure 2).

If $N$ is even, as $a$ and $b$ are antipodal, we can choose $\frac{N-2}{2}$ times $a^{\prime} \in A \cup\{a, b\}$ and $b^{\prime} \in B \cup\{a, b\}$ and use $N-2$ half-colors, so that sets $D_{1}$ and $D_{2}$, but two dipaths connecting $a$ with $b$, are found and colored. At last, one half-color (whose direction can be chosen to ensure the balance condition) is used in this case for the two last dipaths connecting $a$ with $b$.

In the other case, $N$ is odd and we can assume w.l.o.g. that $w(A) \leq\lfloor N / 2\rfloor$ and $w(B) \leq$ $\frac{N-3}{2}$. By choosing $\frac{N-3}{2}$ times $a^{\prime} \in A \cup\{a, b\}$ and $b^{\prime} \in B \cup\{a, b\}$ and using $N-3$ half-colors, we can define and color set $D_{1}$, but four dipaths connecting $a$ and $b$ with one node in $A$, and set $D_{2}$, but two dipaths connecting $a$ with $b$. These last six dipaths can be defined and colored with two more half-colors. From the inductive hypothesis, we can also ensure that the same number of half-colors is used in the two directions of the ring ( $N$ is odd and $M m$ even).

In both cases, we get a colored routing of $(C, w)$ with $h^{+}+h^{-}=M m$ half-colors, so that $h^{+}=\left\lceil\frac{M m}{2}\right\rceil$ and $h^{-}=\left\lfloor\frac{M m}{2}\right\rfloor$.

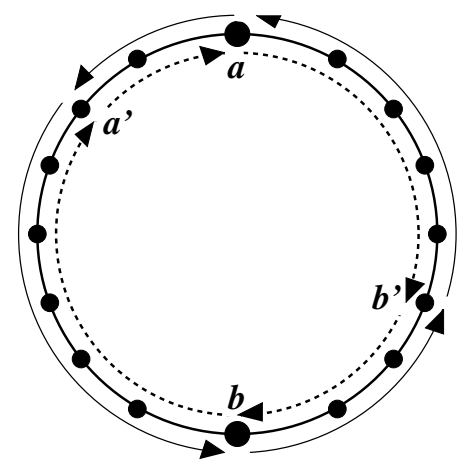

Figure 2: Coloring eight dipaths between $\{a, b\}$ and $\left\{a^{\prime}, b^{\prime}\right\}$ with two half-colors.

As proved in $[5,18]$, we deduce the following result :

Corollary 8 Proposition 5 holds for any weighted ring $\left(C, w_{1}\right)$ with uniform weight equal to 1 .

Proof. The minimum gap of $\left(C, w_{1}\right)$ is at most one, so we are in Case $\mathrm{A}$ and the result follows inductively from the proof of Lemma 7 .

$\mathrm{RR} \mathrm{n}^{\circ} 3700$ 
Corollary 9 Proposition 5 holds for any weighted ring $\left(C, w_{k}\right)$ with uniform weight equal to $k$.

Proof. Let $p$ be the number of nodes of $C$. The load of a tight cut of $\left(C, w_{k}\right)$ is $k^{2}$ times the load of a tight cut of $\left(C, w_{1}\right)$ equal to $\lceil p / 2\rceil\lfloor p / 2\rfloor$. If $p$ is odd or if $p$ and $p / 2$ are even, we can get a solution for $\left(C, w_{k}\right)$ by taking $k^{2}$ times the half-colored routing for $\left(C, w_{1}\right)$, where $h^{+}=h^{-}$. If $p$ is even and $p / 2$ odd, we have $h^{+}=h^{-}+1$ in the half-colored routing for $\left(C, w_{1}\right)$. To ensure the balance condition for $\left(C, w_{k}\right)$, we take $\left\lceil\frac{k^{2}}{2}\right\rceil$ times the half-colored routing for $\left(C, w_{1}\right)$ and $\left\lfloor\frac{k^{2}}{2}\right\rfloor$ times the routing for $\left(C, w_{1}\right)$ where clockwise and anti-clockwise half-colors are swapped.

\section{Case B}

Let $(C, w)$ be a weighted ring with minimum gap $\delta$. We assume that we are not in Case A, so $\delta>1$ and the following condition is satisfied :

Condition B For any two antipodal nodes a and $b$, there exists a tight cut $(S, \bar{S})$ such that $a \in S$ and $b \in S$.

Lemma 10 Any weighted ring satisfying Condition B has uniform weight and an odd number of nodes.

Proof. First notice that for any connected set $X$ of nodes, $w(X)<M$ implies $w(X) \leq m$ and $w(X)>m$ implies $w(X) \geq M$. Note also that any node is antipodal to a least one node.

Let $a, b$ and $d$ be three nodes, such that $b$ and $d$ are adjacent. Let $B$ and $D$ be the two connected sets of nodes of $C-\{a, b, d\}$, between $a$ and $b$, and between $a$ and $d$, respectively.

If $a$ and $b$ are antipodal and if $(S, \bar{S})$ is a tight cut such that $a \in S$ and $b \in S$, then $S=\{a\} \cup B \cup\{b\}$. Indeed, otherwise we have $w(a)+w(B)+w(b)<M$, so $w(a)+w(B)+$ $w(b) \leq m=N-M$ and $w(a)+w(B)+w(b)<N / 2$, but as $a$ and $b$ are antipodal, we have also $w(d)+w(D) \leq\lfloor N / 2\rfloor$ (contradiction).

Condition $\mathrm{B}$ implies that every node has weight at least $\delta$. Indeed, assuming $a$ and $b$ antipodal, we have $w(a)+w(B)+w(b)=M=m+\delta$ and $w(B)+w(b)<M$, so $w(B)+w(b) \leq$ $m$ and $w(a) \geq \delta$. Note also that if node $a$ is antipodal to itself, then $w(a)>N / 2$, there is only one tight cut and we are clearly in Case A.

Assume that both nodes $b$ and $d$ are antipodal with node $a$. Then $w(a)+w(B)+w(b)=M$ and $w(a)+w(D)+w(d)=M$. So $N+w(a)=2 M=M+m+\delta=N+\delta$, hence $w(a)=\delta$.

Assume that $b$ is the only antipodal node with $a$. Then $w(a)+w(B)+w(b)=M$ and $w(B)+w(b) \leq m \leq N / 2$. As $a$ and $d$ are not antipodal and since $w(D)<m \leq N / 2$, we have $w(B)+w(b)>\lfloor N / 2\rfloor$ (contradiction).

Therefore, Condition B implies that every node has at least two antipodal nodes and weight $\delta$. The number of nodes cannot be even, otherwise $\delta=0$ (Case A). So, in Case B the weight is uniform and the number of nodes is odd. 


\section{Proof of Proposition 5}

The proof is made by induction. Let $(C, w)$ be a weighted ring with weight $N$. If $N \leq 1$ then the result is trivial. Assume that Proposition 5 holds for all weighted rings with weight less than $N$. If $(C, w)$ satisfies Condition A, then we use Lemma 7 , else $(C, w)$ has uniform weight and a number of nodes odd, according to Lemma 10, we use then Corollary 9 to conclude.

\section{General case}

Now we extend Proposition 5 (and thus Main Theorem) to the case of weighted trees of rings.

Proposition 11 Let $(\mathcal{T}, w)$ be a weighted tree of rings and $M m$ be the load of a tight cut. Then there exists a routing which can be colored with $h^{+}+h^{-}=M m$ half-colors, with $h^{+}=\left\lceil\frac{M m}{2}\right\rceil$ and $h^{-}=\left\lfloor\frac{M m}{2}\right\rfloor$.

Definition 12 Let $(\mathcal{T}, w)$ be a weighted tree of rings and $P$ a closed Eulerian dipath in $\mathcal{T}$ visiting each node. The interval labelling $I$ for $P$ (see Figure 2) is the assignment of an interval $I(a)$ of integers in $\{1, \ldots, N\}$ to each node a of $\mathcal{T}$, such that :

- distinct nodes are assigned disjoint intervals and each node a is assigned an interval of length $w(a)$;

- for any two integers $i$ and $j$ in $\{1, \ldots, N\}, i<j$, if $i \in I(a)$ and $j \in I(b)$ for some nodes $a$ and $b$, then $a=b$ or $a$ is visited for the first time before $b$ along $P$.

Definition 13 Let $(\mathcal{T}, w)$ be a weighted tree of rings with total weight $N$, let $P$ be a closed Eulerian dipath in $\mathcal{T}$ and let $I$ be the interval labelling for $P$. Two nodes $a$ and $b$ are said to be antipodal if there exist $i \in I(a)$ and $j \in I(b)$ such that $j=i+\left\lfloor\frac{N}{2}\right\rfloor \bmod N$ or $j=i+\left\lceil\frac{N}{2}\right\rceil \bmod N$.

This definition of antipodal nodes of weighted trees of rings is equivalent to that given previously for weighted rings. Also, half-colors can be defined for trees of rings. Note that a closed Eulerian (and Hamilton) dipath $P$ for a weighted tree of rings $(\mathcal{T}, w)$ induces an orientation of each ring of $\mathcal{T}$. Indeed, every arc used by $P$ can be defined clockwise and every other arc can be defined anti-clockwise. We can then call half-color a color used for routing a set of arc-disjoint dipaths using exclusively clockwise or anti-clockwise arcs. Nevertheless, we will need a slightly different definition of half-colors in Case B. Indeed, to achieve Main Theorem it is only required that half-colors can be combined two by two to obtain colors. This will be the case in the proof of Lemmas 17 and 18 where each half-color will be used for routing a set of arc-disjoint dipaths using in each ring exclusively clockwise or anti-clockwise arcs.

As for weighted rings, we first assume that Condition A is satisfied (Case A).

$\mathrm{RR} \mathrm{n}^{\circ} 3700$ 


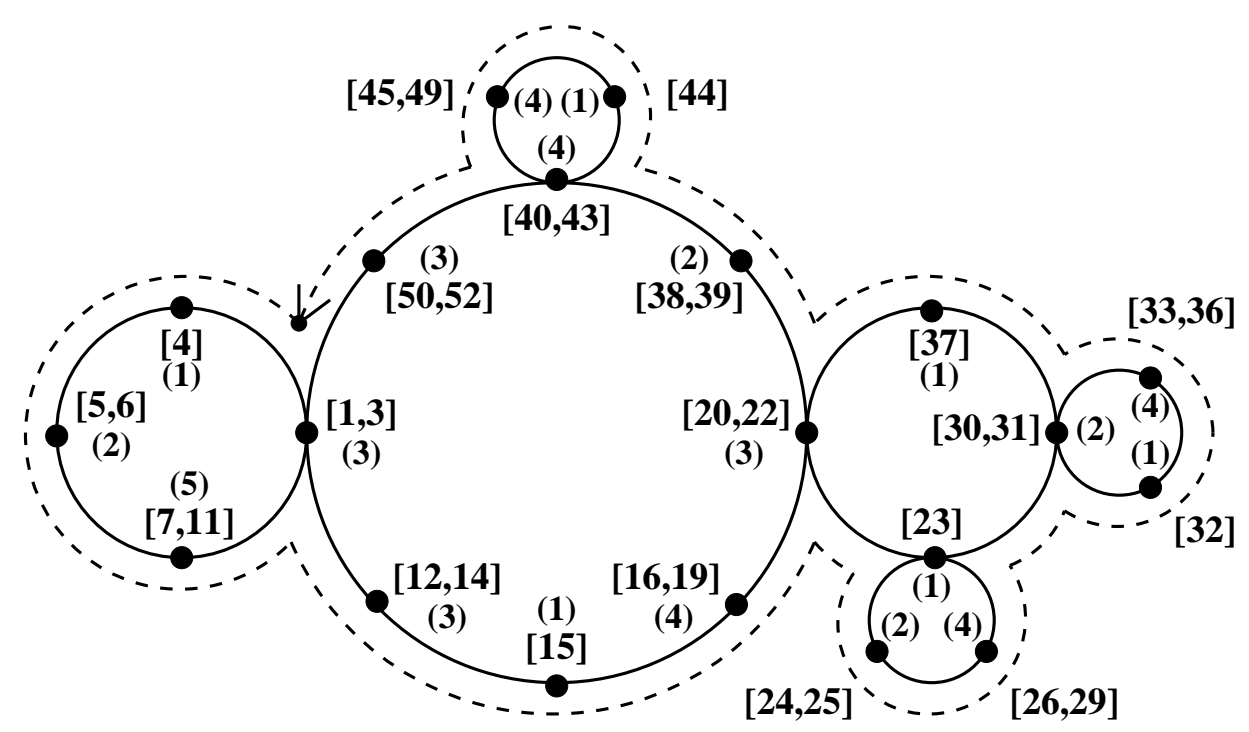

Figure 3: Interval labelling of a weighted tree of rings (weights are in brackets) induced by a closed Eulerian dipath.

Lemma 14 If Proposition 11 holds for all weighted trees of rings with weight less than $N$, then it holds for any weighted tree of rings with weight $N$ satisfying Condition A.

Proof. The same arguments as in the proof of Lemma 7 are used and we give here a short proof. Given a weighted tree of rings $(\mathcal{T}, w)$, let $P$ be a closed Eulerian dipath in $\mathcal{T}$. We consider two antipodal nodes $a$ and $b$ and we can assume w.l.o.g. that $a$ is visited for the first time before $b$ along $P$. Let $A$ be the set of nodes visited after $a$ and before $b$ and let $B$ be the set of nodes visited before $a$ or after $b$. By defining $A$ and $B$ in this manner and by routing all requests clockwise or anti-clockwise along $P$, the proof of Lemma 7 can be adapted and leads to the same result. $\square$

Let $(\mathcal{T}, w)$ be a weighted tree of rings with minimum gap $\delta$. If Condition $\mathrm{A}$ is not satisfied, then $\delta>1$ and Condition B holds (Case B).

Definition $15 A$ main ring of $(\mathcal{T}, w)$ is any ring where some tight cut occurs. A block of a main ring $\mathcal{C}$ is any set of nodes remaining connected after removing all the arcs of $\mathcal{C}$.

Whereas for weighted rings Condition B implies an odd number of nodes of the same weight, for weighted trees of rings we need to add the condition that every block of a main ring has weight at most $N / 2$ (Case $\mathrm{B} 1$ ). 
Lemma 16 Consider a weighted tree of rings satisfying Condition $B$ and having a main ring with blocks all of weight at most N/2. Then there is an odd number of blocks all of the same weight.

Sketch of proof. See the proof of Lemma 10. (Blocks of a tree of rings correspond to nodes of a ring and antipodal blocks are defined as containing antipodal nodes.)

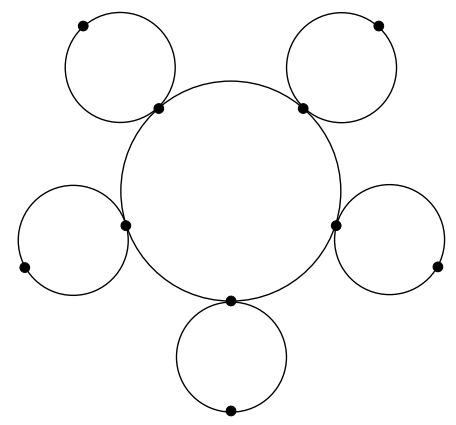

Figure 4: A weighted tree of rings in Case B1. All weights are equal to 1.

The remaining case to study is that of a weighted tree of rings satisfying Condition $\mathrm{B}$ and having a main ring with one block of weight greater than $N / 2$ (Case B2). So there is exactly one tight cut in the main ring separating this block from the other blocks. Moreover Condition B implies the existence of another tight cut out of the main ring, as two antipodal nodes can be chosen respectively in and out of the main ring. So we can see the weighted tree of rings as the union of weighted trees of rings (at least two of them having the same maximum weight) which intersect two by two in the same weighted node.

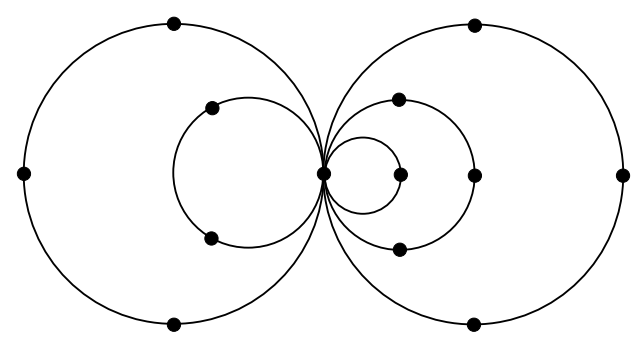

Figure 5: A weighted tree of rings in Case B2. All weights are equal to 1.

In order to complete the proof of Main Theorem we need to solve Case B1 and Case B2.

Lemma 17 Proposition 11 holds for any weighted tree of rings $(\mathcal{T}, w)$ with $2 p+1$ blocks $(p>0)$ of weight $k$.

$\mathrm{RR} \mathrm{n}^{\circ} 3700$ 
Proof. The load of a tight cut is $p(p+1) k^{2}$. The half-coloring of $(\mathcal{T}, w)$ follows from the solution obtained for the ring $\left(C_{2 p+1}, w_{1}\right)$ with $2 p+1$ nodes and uniform unit weight, that uses $p(p+1)$ half-colors.

We number blocks of $\mathcal{T}$ with integers from 1 to $2 p+1$ like the nodes of $C_{2 p+1}$ and for each integer $i$ we take a closed Eulerian dipath $P_{i}$ of block number $i$ and its interval labelling $I_{i}$. Moreover, for $1 \leq i \leq 2 p+1$ and $1 \leq x \leq k$, we denote by $l_{i}(x)$ the node of block number $i$ such that $x \in I_{i}\left(l_{i}(x)\right)$. We establish a correspondence between each half-color used in a solution for $\left(C_{2 p+1}, w_{1}\right)$ and a set of $k^{2}$ half-colors for $(\mathcal{T}, w)$. Every dipath in $\mathcal{T}$ will be routed in every block along its Eulerian dipath.

Assume that $c$ is a half-color used in $\left(C_{2 p+1}, w_{1}\right)$ for clockwise dipaths. For each pair of integers $x$ and $y$ such that $1 \leq x, y \leq k$, we use in $(\mathcal{T}, w)$ a half-color to color dipaths 1$)$ from node $l_{i}(x)$ to node $l_{i}(y)$ for each $i$, and 2) from node $l_{i}(y)$ to node $l_{j}(x)$ if there is in $\left(C_{2 p+1}, w_{1}\right)$ a dipath from node $i$ to node $j$ colored by $c$. Note that these dipaths are defined clockwise in the main ring, clockwise in the blocks if $x \leq y$ and anti-clockwise in the blocks if $y<x$.

Similarly, if $c$ is used in $\left(C_{2 p+1}, w_{1}\right)$ for anti-clockwise dipaths then for $1 \leq x, y \leq k$ we use in $(\mathcal{T}, w)$ a half-color to color dipaths 1$)$ from node $l_{i}(y)$ to node $l_{i}(x)$ for each $i$, and 2) from node $l_{i}(x)$ to node $l_{j}(y)$ if there is in $\left(C_{2 p+1}, w_{1}\right)$ a dipath from node $i$ to node $j$ colored by $c$. Note that these dipaths are defined anti-clockwise in the main ring, anti-clockwise in the blocks if $x \leq y$ and clockwise in the blocks if $y<x$.

This symmetric assignment ensures that half-colors can be combined two by two to obtain colors, so Proposition 11 holds in Case B1.

Lemma 18 Proposition 11 holds for any weighted tree of rings $(\mathcal{T}, w)$ that is the union of weighted trees of rings (at least two of them having the same maximum weight) which intersect two by two in the same weighted node.

Sketch of proof. Let $v$ be the common weighted node of the weighted subtrees of rings whose union forms $(\mathcal{T}, w)$. Note that $v$ is at the border of every tight cut. In each subtree of rings we take a closed Eulerian dipath and every dipath in $\mathcal{T}$ will be routed in every subtree of rings along its Eulerian dipath. A good routing strategy is to combine the different routings for each subtree of rings obtained by adding to the weight of node $v$ the weights of the other subtrees of rings (Condition A is then satisfied in each so modified subtree of rings). The main problem consists in coloring the set of all dipaths going to or from or through $v$. This is obviously equivalent to color a set of dipaths in a subdivided star, i.e. in a graph that is the union of paths (twice more than subtrees of rings) which intersect two by two in the same node. Theorem 21 of [4] states the existence of a coloring in this case with the right number of colors. The remaining dipaths go neither to neither from nor through $v$ and can be colored more easily. 


\section{References}

[1] A. Aggarwal, A. Bar-Noy, D. Coppersmith, R. Ramaswami, B. Schieber, and M. Sudan. Efficient routing and scheduling algorithms for optical networks. In Proc. of the 5th Ann. ACM-SIAM Symp. on Discrete Algorithms (SODA'94), pages 412-423. ACM Press, January 1994.

[2] Y. Aumann and Y. Rabani. Improved bounds for all optical routing. In Proc. of the 6th Ann. ACM-SIAM Symp. on Discrete Algorithms (SODA'95), pages 567-576. ACM Press, January 1995.

[3] B. Beauquier. All-to-all communication in some wavelength-routed all-optical networks. Networks (Wiley-Interscience), 33(3):179-187, May 1999.

[4] B. Beauquier, J-C. Bermond, L. Gargano, P. Hell, S. Pérennes, and U. Vaccaro. Graph problems arising from wavelength-routing in all-optical networks. In Proc. of 2nd IEEE Workshop on Optics and Computer Science (WOCS, part of IPPS'97). IEEE Press, April 1997.

[5] J.-C. Bermond, L. Gargano, S. Pérennes, A.A. Rescigno, and U. Vaccaro. Efficient collective communication in optical networks. In Proc. of 23rd Int. Colloq. on Automata, Languages and Programming (ICALP'96), volume 1099 of Lecture Notes in Computer Science, pages 574-585. Springer-Verlag, July 1996.

[6] N. K. Cheung, Nosu K., and G. Winzer. Special issue on dense WDM networks. Journal on Selected Areas in Communications, 8, 1990.

[7] T. Erlebach and K Jansen. Scheduling of virtual connections in fast networks. In Proc. of Parallel Systems and Algorithms (PASA), pages 13-32, 1996.

[8] L. Gargano, P. Hell, and S. Pérennes. Colouring paths in directed symmetric trees with applications to WDM routing. In Proc. of 24th Int. Colloq. on Automata, Languages and Programming (ICALP'g7), volume 1256 of Lecture Notes in Computer Science, pages 505-515. Springer-Verlag, July 1997.

[9] P. E. Green. The future of fiber-optic computer networks. IEEE Computer, 24(9):7887, September 1991.

[10] P. E. Green. Fiber-Optic Communication Networks. Prentice-Hall, 1993.

[11] C. Kaklamanis and P. Persiano. Efficient wavelength routing on directed fiber trees. In Proc. of European Symp. on Algorithms (ESA'96), volume 1136 of Lecture Notes in Computer Science, pages 460-470, 1996.

[12] M. Mihail, C. Kaklamanis, and S. Rao. Efficient access to optical bandwidthwavelength routing on directed fiber trees, rings, and trees of rings. In Proc. of 36th Ann. Symp. on Foundations of Computer Science (FOCS'95), pages 548-557. IEEE, October 1995. 
[13] D. Minoli. Telecommunications Technology Handbook. Artech House, 1991.

[14] B. Mukherjee. WDM-based local lightwave networks, Part 1: Single-hop systems. IEEE Network Magazine, 6(3):12-27, May 1992.

[15] P. Raghavan and E. Upfal. Efficient routing in all-optical networks. In Proc. of the 26th Ann. ACM Symp. on Theory of Computing (STOC'94), pages 134-143, May 1994.

[16] R. Ramaswami. Multiwavelength lightwave networks for computer communication. IEEE Communications Magazine, 31(2):78-88, February 1993.

[17] O. Togni. Optical all-to-all communication in inflated networks. In Proc. of the 5th Ann. Int. Workshop on Graph-Theoretic Concepts in Computer Science (WG'98), volume 1517 of Lecture Notes in Computer Science, pages 78-87. Springer-Verlag, 1998.

[18] G. Wilfong. Minimizing wavelengths in an all-optical ring network. In Proc. of the 7th Int. Symp. on Algorithms and Computation (ISAAC'96), volume 1178 of Lecture Notes in Computer Science, pages 346-355. Springer-Verlag, 1996. 


\section{Contents}

1 Introduction and Motivation $\quad 3$

2 Preliminaries 4

3 Weighted rings $\quad 6$

4 General case $\quad 9$

$\mathrm{RR} \mathrm{n}^{\circ} 3700$ 


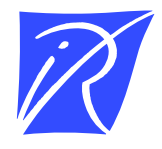

Unité de recherche INRIA Sophia Antipolis 2004, route des Lucioles - B.P. 93 - 06902 Sophia Antipolis Cedex (France)

Unité de recherche INRIA Lorraine : Technopôle de Nancy-Brabois - Campus scientifique 615, rue du Jardin Botanique - B.P. 101 - 54602 Villers lès Nancy Cedex (France)

Unité de recherche INRIA Rennes : IRISA, Campus universitaire de Beaulieu - 35042 Rennes Cedex (France)

Unité de recherche INRIA Rhône-Alpes : 655, avenue de l'Europe - 38330 Montbonnot St Martin (France)

Unité de recherche INRIA Rocquencourt : Domaine de Voluceau - Rocquencourt - B.P. 105 - 78153 Le Chesnay Cedex (France)

Éditeur

INRIA - Domaine de Voluceau - Rocquencourt, B.P. 105 - 78153 Le Chesnay Cedex (France)

http://www.inria.fr

ISSN 0249-6399 